\title{
A Crossed Sliding Luttinger Liquid Phase
}

\author{
Ranjan Mukhopadhyay, C.L. Kane, and T.C. Lubensky \\ Dept. of Physics, Univ. of Penn., Philadelphia, PA 19104
}

(October 25, 2018)

\begin{abstract}
We study a system of crossed spin-gapped and gapless Luttinger liquids. We establish the existence of a stable non-Fermi liquid state with a finite-temperature, long-wavelength, isotropic electric conductivity that diverges as a power law in temperature $T$ as $T \rightarrow 0$. This two-dimensional system has many properties characteristic of a true isotropic Luttinger liquid, though at zero temperature it becomes anisotropic. This model can easily be extended to three dimensions.

PACS numbers: 71.10.Hf, 71.10.Pm, 74.22.Mn
\end{abstract}

For over two decades a central theme in the study of correlated electronic systems has been the drive to understand and classify electronic states that do not conform to Landau's Fermi-liquid theory. A clear example of such "non-Fermi liquid" physics occurs in one dimension (1D) [1], where arbitrarily weak interactions destroy the Fermi surface and invalidate the notion of independent quasiparticles at low energy. Away from charge-density-wave instabilities, the interacting 1D electron gas forms a Luttinger liquid in which the discontinuity in occupation at the Fermi energy of a normal Fermi liquid is replaced by a power-law singularity, and the low-lying excitations are bosonic collective modes in which spin and charge decouple.

Following the discovery of high-temperature superconductivity, Anderson suggested that the unusual normalstate properties of the cuprates were the result of similar non-Fermi-liquid physics in two dimensions [2]. However, the study of non-Fermi liquids in higher dimensions has proven to be quite difficult. Since the Fermi liquid is stable for weak interactions, perturbative methods fail [3]. Moreover, generalizations of the bosonization technique to isotropic systems in higher dimensions have indicated that Fermi liquid theory survives provided the interactions are not pathologically long ranged [4]. An alternative approach has been to study anisotropic systems consisting of arrays of parallel weakly coupled $1 \mathrm{D}$ wires [5]. It has recently been proposed [6, 7] that for a range of interwire charge and current interactions, there is a smectic-metal (SM) phase in which Josephson, chargeand spin-density-wave, and single-particle couplings are irrelevant. This phase is an anistropic sliding Luttinger liquid phase whose transport properties exhibit powerlaw singularities like those of a 1D Luttinger liquid. It is the quantum analog of the sliding phases of coupled classical XY models found by O'Hern et al. [8,9].

We consider a square network of $1 \mathrm{D}$ wires formed by coupling two perpendicular smectic metals [10] and show that it exhibits a new crossed sliding Luttinger Liquid (CSLL) phase. We establish a range of couplings for which both this phase and the anisotropic twodimensional smectic-metal phase from which it is constructed are stable with respect to a large class of operators. At finite temperature $T$, the CSLL phase is an isotropic $2 D$ Luttinger liquid with an isotropic longwavelength conductivity that diverges as a power-law in $T$ as $T \rightarrow 0$. At $T=0$, it is essentially two independent smectic metals. This model could be realized in manmade strucures constructed from quantum wires such as carbon nanotubes. Extension of the model to a threedimensional stack may be relevant to the stripe phases of the cuprates. Based on neutron and x-ray scattering measurements, it has been suggested that spin-charge stripes in the adjacent $\mathrm{CuO}_{2}$ plane are orthogonal to each other [1].

The Lagrangian density describing the low-energy behavior of a one-dimensional Luttinger liquid is

$$
\mathcal{L}_{0}=\frac{1}{2} \kappa\left[v^{-1}\left(\partial_{\tau} \phi\right)^{2}+v\left(\partial_{x} \phi\right)^{2}\right]
$$

where $\phi$ is a bosonic field [12] and $\kappa$ and the sound velocity, $v$, are non-universal functions of the coupling constants. For repulsive interactions, $\kappa>1$. The Lagrangian density in terms of the dual phase variable $\theta$ has the same form as Eq. (11), but with $v$ replaced by $1 / v$. For spin$1 / 2$ fermions, the spin excitations could either be gapped or gapless. In the spin-gap, Luther-Emery regime, the system can by described by a single Luttinger liquid for charge. In the gapless case, both spin and charge are dynamical degrees of freedom, and there are two Luttinger parameters $\left(\kappa_{c}, \kappa_{s}\right)$, and two velocities $\left(v_{c}, v_{s}\right)$.

Now consider a two-dimensional array of parallel quantum wires. To begin with, we consider the spin gapped case, so that the spin fluctuations on each wire are effectively frozen out at low energies. It has been suggested that this case might describe the stripe phases of high-temperature superconductors 13 . In general, we expect a generalized current-current interaction between the wires, which can be represented by a Lagrangian density of the form

$$
\mathcal{L}_{\mathrm{int}}=\frac{1}{2} \sum_{n, n^{\prime}, \mu} j_{\mu, n}(x, \tau) \tilde{W}_{\mu}\left(n-n^{\prime}\right) j_{\mu, n^{\prime}}(x, \tau),
$$

where $j_{\mu, n}=\left(\rho_{n}(x, \tau), J_{n}(x, \tau)\right)$ with $\rho_{n}=\partial_{x} \phi_{n}(x, \tau)$ the density and $J_{n}=\partial_{\tau} \phi_{n}(x, \tau)$ the current on the $n$th wire. This interaction is marginal and should be included 
in the fixed-point action. It is invariant under the "sliding" transformations $\phi_{n} \rightarrow \phi_{n}+\alpha_{n}$ and $\theta_{n} \rightarrow \theta_{n}+\alpha_{n}^{\prime}$. Equations (11) and (2) define the fixed-point action of the smectic-metal phase [6], which can be written in Fourier space as

$$
S=\sum_{Q} \frac{1}{2}\left\{W_{0}\left(q_{\perp}\right) \omega^{2}+W_{1}\left(q_{\perp}\right) q_{\|}^{2}\right\}|\phi(Q)|^{2},
$$

where $Q=\left(\omega, q_{\|}, q_{\perp}\right)$, with $q_{\|}$the momentum along the chain and $q_{\perp}$ perpendicular to the chains.

We can study perturbatively the relevance of various operators to ascertain the stability of the smectic-metal (SM) phase. Due to the spin gap, single-particle hopping between chains is irrelevant, and the only inter-chain interactions involving only pairs of chains that could become relevant are the Josephson (SC) and CDW couplings, whose respective Hamiltonian densities are

$$
\begin{aligned}
\mathcal{H}_{\mathrm{SC}, n} & =\sum_{i} \mathcal{J}_{n} \cos \left[\sqrt{2 \pi}\left(\theta_{i}-\theta_{i+n}\right)\right], \\
\mathcal{H}_{\mathrm{CDW}, n} & =\sum_{i} \mathcal{V}_{n} \cos \left[\sqrt{2 \pi}\left(\phi_{i}-\phi_{i+n}\right)\right],
\end{aligned}
$$

where $\mathcal{J}_{n}$ are the inter-chain Josephson couplings and $\mathcal{V}_{n}$ the inter-chain particle-hole (CDW) interactions. The scaling dimensions of $\mathcal{H}_{\mathrm{SC}, n}$ and $\mathcal{H}_{\mathrm{CDW}, n}$ are, respectiveley,

$$
\begin{aligned}
\Delta_{\mathrm{SC}, n} & =\int_{-\pi}^{\pi} \frac{d q_{\perp}}{2 \pi}\left(1-\cos n q_{\perp}\right) \kappa\left(q_{\perp}\right), \\
\Delta_{\mathrm{CDW}, n} & =\int_{-\pi}^{\pi} \frac{d q_{\perp}}{2 \pi} \frac{\left(1-\cos n q_{\perp}\right)}{\kappa\left(q_{\perp}\right)}
\end{aligned}
$$

where $\kappa\left(q_{\perp}\right)=\sqrt{W_{0}\left(q_{\perp}\right) W_{1}\left(q_{\perp}\right)}$. For the smectic-metal phase to be stable, these perturbations should be irrelevant, which implies

$$
\Delta_{\mathrm{CDW}, n}>2, \quad \Delta_{\mathrm{SC}, n^{\prime}}>2
$$

for all $n$ and $n^{\prime}$. In addition to the pairwise operators of Eq. (4), there are multiwire operators of the form $\mathcal{H}_{\mathrm{CDW},\left\{\sigma_{n}\right\}}=\sum_{i} \mathcal{T}\left(\sigma_{n}\right) \cos \left[\sqrt{2 \pi}\left(\sum_{n} \sigma_{n} \theta_{i+n}\right)\right]$ where the $\sigma_{n}^{\prime} \mathrm{s}$ are integers satisfying $\sum \sigma_{n}=0$. The overall strengths of these interactions measured by $\mathcal{T}\left(\sigma_{n}\right)$ are much smaller than those of $\mathcal{H}_{\mathrm{CDW}, n}$, and they become important only at very small temperatures even if they are relevant. We will therefore ignore them in this article, delaying a more complete study of their effects to a future publication.

To explore the regions of stability of the SM phase, we follow refs. [7],9] and take

$$
\kappa\left(q_{\perp}\right)=K\left[1+\lambda_{1} \cos \left(q_{\perp}\right)+\lambda_{2} \cos \left(2 q_{\perp}\right)\right] .
$$

We define $\Delta_{\mathrm{SC}, n}=a_{n} K$ and $\Delta_{\mathrm{CDW}, n}=b_{n} / K$, where $a_{1}=\left(1-\lambda_{1} / 2\right), a_{2}=\left(1-\lambda_{2} / 2\right)$ and $a_{n}=1$ for $n \neq 1,2$. The SM phase becomes unstable to inter-chain Josephson couplings for $K$ less than $K_{\mathrm{SC}}=\max _{n}\left(2 / a_{n}\right)$ and unstable to inter-chain CDW interactions for $K$ greater than $K_{\mathrm{CDW}}=\min _{n}\left(b_{n} / 2\right)$. Thus the smectic metal phase is stable with respect to pairwise interactions over a window of $K, K_{\mathrm{SC}}<K<K_{\mathrm{CDW}}$, provided

$$
\beta \equiv \frac{K_{\mathrm{CDW}}}{K_{\mathrm{SC}}}=\left.\frac{a_{n} b_{m}}{4}\right|_{\text {min.wrt.m\&n }}>1 .
$$

If $\beta<1$, the system goes directly from a $2 \mathrm{D}$ superconducting (SC) phase to a CDW crystal as $K$ is increased, without passing through the SM phase. $\kappa\left(q_{\perp}\right)$ reaches a minimum, $\kappa\left(k_{0}\right) \equiv \Delta K$ at some $q_{\perp}=k_{0}$. The SM phase is stabilised at small $\Delta$, that is, when the system is close to a CDW instability in which there is a periodic modulation of charge on different wires. Setting $\Delta=10^{-5}$, we plot $\beta$ as a function of $k_{0}$. We note that there are regions of stability of the smectic phase with respect to $\mathcal{H}_{\mathrm{SC}}$ and $\mathcal{H}_{\mathrm{CDW}}$, for positive as well as negative values of $\lambda_{1}$.

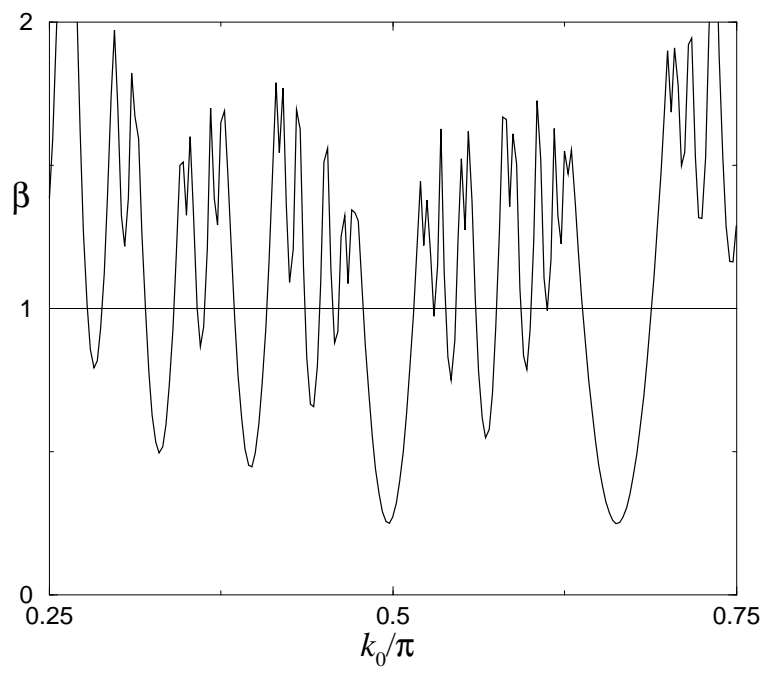

FIG. 1. Plot of $\beta \equiv K_{C D W} / K_{S C}$ as a function of of $k_{0} / \pi$. For $\beta>1$ there exists a region of $K$ over which the non-Fermi liquid phase is stable to all pairwise interactions.

We consider next a square grid of wires, again for the spin-gapped case. There are two arrays of quantum wires, the $X$ - and $Y$ - arrays running, respectivley, parallel to the $x$ - and $y$-directions. Each wire now sees a periodic one-electron potential from the array of wires crossing it that leads to a new band structure with new band gaps. We assume that the Fermi surface is between gaps so that the wires would be conductors in the absence of further interactions. By removing degrees of freedom with wavelengths smaller than the inverse wire separation, we obtain a new effective theory whose form is identical to the theory before the periodic potential was introduced. Thus, in the absence of two-particle interactions between crossed arrays, the system could be in a phase consisting of two crossed, non-interacting smecticmetal states. 
We will now demonstrate that the sliding phase in a crossed grid is stable if the sliding phase in the component planar arrays is stable. In addition to the interwire couplings within each array, we need to consider Coulomb interactions between wires on the $\mathrm{X}$-array and wires on the Y-array. These inter-array couplings are marginal and should be included in the fixed point. They do not, however, change the dimensions of the operators, except by renormalizing $\kappa\left(q_{\perp}\right)$. For a stable sliding phase, additional interactions between the two arrays, such as the Josephson and CDW couplings, have to be irrelevant. We will show that it is possible to tune $\kappa\left(q_{\perp}\right)$ such that this is indeed the case.

The Coulomb interactions between electrons on intersecting wires gives rise to a term in the Hamiltonian of the form $V_{m, n}^{c}(x, y) \rho_{x, m}(x) \rho_{y, n}(y)$, where $\rho_{x, m}(x)\left[\rho_{y, m}(y)\right]$ is the electron density on the $m$ th wire on the $X(Y)$-array at position $x(y)$. We expect $V_{m, n}^{c}(x, y)$ to have the form $V^{c}(x-n a, y-m a)$, where $a$ is the distance between parallel wires. If all parameters for the $X$ and $Y$-arrays are the same, the crossed-grid action as a functional of the $\theta$ and $\phi$ variabless can be written as

$$
\begin{aligned}
S= & \frac{1}{2} \int \frac{d \omega d q_{x} d q_{y}}{(2 \pi)^{3}}\left[V^{\theta}\left(q_{y}\right) q_{x}^{2}\left|\theta_{x}\right|^{2}+V^{\theta}\left(k_{x}\right) q_{y}^{2}\left|\theta_{y}\right|^{2}\right. \\
& +V^{\phi}\left(q_{y}\right) q_{x}^{2}\left|\phi_{x}\right|^{2}+V^{\phi}\left(q_{x}\right) q_{y}^{2}\left|\phi_{y}\right|^{2} \\
& +V^{C}\left(q_{x}, q_{y}\right) q_{x} q_{y}\left\{\phi_{x} \phi_{y}^{*}+\text { c.c. }\right\} \\
& \left.-i \omega q_{x}\left\{\theta_{x}^{*} \phi_{x}+\text { c.c. }\right\}-i \omega q_{y}\left\{\theta_{y}^{*} \phi_{y}+\text { c.c. }\right\}\right]
\end{aligned}
$$

with obvious definitions for $\phi_{x}=\phi_{x}\left(\omega, q_{x}, q_{y}\right), \phi_{y}, \theta_{x}$, and $\theta_{y}$. It should be noted that this is an effective theory with $-\frac{\pi}{a}<q_{x}, q_{y}<\frac{\pi}{a}$. Integrating out the $\phi$ variables, we are left with an effective action

$$
\begin{aligned}
S_{\theta}= & \frac{1}{2} \int \frac{d \omega d q_{x} d q_{y}}{(2 \pi)^{3}}\left[\frac{1}{\kappa_{x}(\mathbf{q})}\left(v_{x}(\mathbf{q}) q_{x}^{2}+\frac{\omega^{2}}{v_{x}(\mathbf{q})}\right)\left|\theta_{x}\right|^{2}\right. \\
& +\frac{1}{\kappa_{y}(\mathbf{q})}\left(v_{y}(\mathbf{q}) q_{y}^{2}+\frac{\omega^{2}}{v_{y}(\mathbf{q})}\right)\left|\theta_{y}\right|^{2} \\
& \left.+V_{R}^{c}(\mathbf{q}) \omega^{2}\left\{\theta_{x} \theta_{y}^{*}+\text { c.c. }\right\}\right]
\end{aligned}
$$

where $\kappa_{x}(\mathbf{q})=\sqrt{\frac{\gamma(\mathbf{q})}{V^{\phi}\left(q_{x}\right) V^{\theta}\left(q_{y}\right)}}, \quad v_{x}(\mathbf{q})=\sqrt{\frac{V^{\theta}\left(q_{y}\right) \gamma(\mathbf{q})}{V^{\phi}\left(q_{x}\right)}}$ with $\gamma(\mathbf{q})=V^{\phi}\left(q_{x}\right) V^{\phi}\left(q_{y}\right)-\left(V^{c}(\mathbf{q})\right)^{2}$, and $\kappa_{y}(\mathbf{q})=$ $\kappa_{x}(P \mathbf{K}) ; \quad v_{y}(\mathbf{q})=v_{x}(P \mathbf{q})$ where $P \mathbf{q}=P\left(q_{x}, q_{y}\right)=$ $\left(q_{y}, q_{x}\right)$. Correlation functions for $\theta_{x}$ and $\theta_{y}$ can be calculated directly from Eq. (10). $\theta_{x}-\theta_{y}$ cross-correlations are non-singular, whereas, $\theta_{x}-\theta_{x}$ and $\theta_{y}-\theta_{y}$ correlations have singular parts with exactly the same functional forms as they have in the absence of coupling between layers, but with the $\kappa(q)$ function in expressions for the scaling exponents replaced by

$$
\kappa\left(q_{\perp}\right)=\kappa_{x}\left(0, q_{\perp}\right)=\kappa_{y}\left(q_{\perp}, 0\right) .
$$

Thus, other than renormalizing $\kappa(q)$, the coupling $V_{m, n}^{c}$ between the two arrays leaves the dimensions of all operators unchanged. Equations (10) and (11) define a 2D
non-Fermi liquid with scaling properties to be discussed below.

First, however, we must verify that it is possible to choose potentials so that this $2 \mathrm{D}$ non-Fermi liquid is stable with respect to perturbations. All pairwise couplings within a given array, i.e. $\mathcal{H}_{\mathrm{SC}, n}^{X}, \mathcal{H}_{\mathrm{CDW}, n}^{X}, \mathcal{H}_{\mathrm{SC}, n}^{Y}$ and $\mathcal{H}_{\mathrm{CDW}, n}^{Y}$ defined as obvious generalizations of Eq. (4), can be rendered irrelevant by choosing $\kappa\left(q_{\perp}\right)$ as in the case of an individual array. We must also consider Josephson and CDW couplings between the two arrays, which operate at the points of crossing $(x, y)=(n a, m a)$ of wire $m$ in the $X$-array and wire $n$ of the $Y$-array and, respectively, take the form

$$
\begin{aligned}
\mathcal{H}_{\mathrm{SC}}^{X Y} & =\mathcal{J}^{X Y} \cos \left[\sqrt{2 \pi}\left(\theta_{x, m}(n a)-\theta_{y, n}(m a)\right)\right] \\
H_{\mathrm{CDW}}^{X Y} & =\mathcal{V}^{X Y} \cos \left[\sqrt{2 \pi}\left(\phi_{x, m}(n a)-\phi_{y, n}(m a)\right)\right] .
\end{aligned}
$$

The dimensions of these operators are, respectively,

$$
\begin{aligned}
\Delta_{\mathrm{SC}, \infty} & \equiv \int_{-\pi}^{\pi} \frac{d q}{2 \pi} \kappa(q)=K \\
\Delta_{\mathrm{CDW}, \infty} & \equiv \int_{-\pi}^{\pi} \frac{d q}{2 \pi} \frac{1}{\kappa(q)} \simeq \frac{1}{K} \frac{1}{\sqrt{C \Delta}}
\end{aligned}
$$

where we assume that $\kappa(q)$ has the form given by Eq. (7), $\Delta$ is defined as before, and $C \equiv \kappa^{\prime \prime}\left(k_{0}\right) / 2 K$. If $\kappa$ is chosen such that Eq. (7) is satisfied for each array, then $\mathcal{H}_{\mathrm{SC}}^{X Y}$ and $\mathcal{H}_{\mathrm{CDW}}^{X Y}$ are automatically irrelevant. We do not need any further fine tuning of $\kappa$ to get this $2 \mathrm{D}$ sliding Luttinger liquid phase, and there is a stable CSLL phase.

We now investigate the transport properties of the CSLL phase. The conductivities of an array of parallel wires has been considered by Emery et. al [6]. In the presence of impurities, the resistivity along the wires, $\rho_{\|}$, vanishes as $T^{\alpha_{\|}}[1]$, with $\alpha_{\|}=\Delta_{\mathrm{CDW}, \infty}-2$. The perpendicular conductivity, $\sigma_{\perp}$, goes as $T^{\alpha_{\perp}}$ with $\alpha_{\perp}=2 \Delta_{\mathrm{SC}}-3$, where $\Delta_{\mathrm{SC}}$ is the minimum of $\Delta_{\mathrm{SC}, 1}$ and $\Delta_{\mathrm{SC}, 2}$. The conductance, $\sigma_{c}$, arising from the Josephson coupling at the contact beteen the crossed wires satisfies $\sigma_{c} \sim T^{\alpha_{c}}$, where $\alpha_{c}=2 \Delta_{\mathrm{SC}, \infty}-3$.

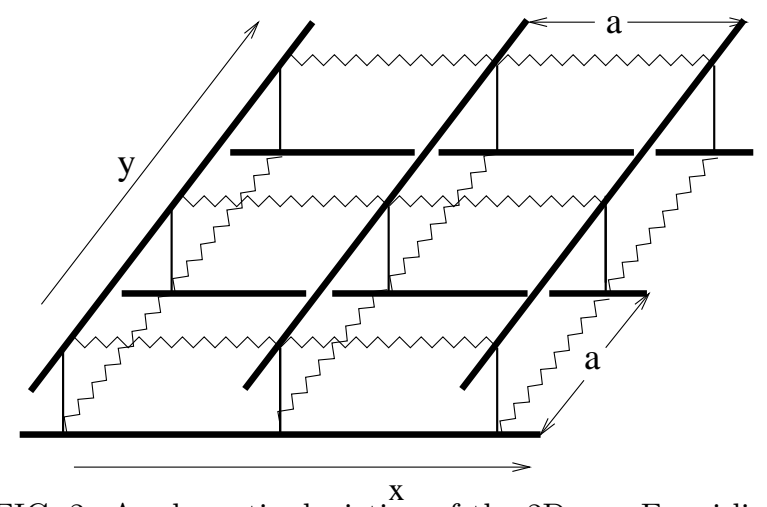

FIG. 2. A schematic depiction of the $2 \mathrm{D}$ non-Fermi liquid as a resistor network, with two parallel arrays of wire running along the $x$ and $y$-axes, with nodes in the $z$ direction 
Thus we can model our 2D non-Fermi liquid as the resistor network depicted in Fig. (2) with nodes at the vertical Josephson junctions between the arrays at $r_{m n}$. The nodes of the $X(Y)$-array are connected by nearest neighbor resistors with conductances $\sigma_{\|}=\rho_{\|}^{-1}$ if they are parallel to the $x(y)$-axis and $\sigma_{\perp}$ if they are perpendicular to the $x$-axis( $y$-axis). Nearest neighbor nodes of the $X$ and $Y$-arrays are connected by resistors of conductance $\sigma_{c}$. In the continuum limit, the 2D current densities in the plane of the $\alpha$ grids $(\alpha=X, Y)$ is $J_{i}^{\alpha}=\sigma_{i j}^{\alpha} E_{j}$ where $\sigma_{i j}^{X}=\sigma_{\|} e_{x i} e_{x j}+\sigma_{\perp} e_{y i} e_{y j}$ and $\sigma_{i j}^{Y}=\sigma_{\perp} e_{x i} e_{x j}+\sigma_{\|} e_{y i} e_{y j}$ and $\mathbf{E}$ is the in-plane electric field. The current per unit area passing between the planes is $J_{n}=\left(\sigma_{c} / a^{2}\right)\left(V^{X}-V^{Y}\right)$ where $V$ is the local voltage. In this limit, the local voltages satisfy

$$
\begin{gathered}
-\sigma_{i j}^{X} \partial_{i} \partial_{j} V^{X}+\frac{\sigma_{c}}{a^{2}}\left(V^{X}-V^{Y}\right)=\mathcal{T}^{X} \\
-\sigma_{i j}^{Y} \partial_{i} \partial_{j} V^{Y}-\frac{\sigma_{c}}{a^{2}}\left(V^{X}-V^{Y}\right)=\mathcal{T}^{Y}
\end{gathered}
$$

where $\mathcal{T}^{X}$ and $\mathcal{T}^{Y}$ are current densities (current/area) injected, respectively, into the $X$ and $Y$-grids. If no currents are injected, then this equation is solved by $V^{X}=V^{Y}=-\mathbf{E} \cdot \mathbf{x}$ to produce a total in-planar curent density

$$
J_{i} \equiv J_{i}^{X}+J_{i}^{Y}=\left(\sigma_{i j}^{X}+\sigma_{i j}^{Y}\right) E_{j}=\left(\sigma_{\|}+\sigma_{\perp}\right) E_{i} .
$$

Thus under a uniform electric field, the double layer behaves like an isotropic 2D material with in-plane conductivity $\sigma=\sigma_{\|}+\sigma_{\perp} \simeq \sigma_{\|}$, or equivalently with an isotropic resistivity that vanishes as $\rho_{\|} \sim T^{\alpha_{\|}}$. If currents are spatially nonuniform, as they are, for example, when current is inserted at one point and extracted from another, there is a crossover from isotropic to anisotropic behavior at length scales less than $l=a \sqrt{\frac{\sigma_{\|}+\sigma_{\perp}}{\sigma_{c}}} \sim T^{-\left(\alpha_{\|}+\alpha_{c}\right) / 2}$ that diverges as $T \rightarrow 0$.

This two-layer CSLL model can, quite simply, be extended to three dimensions by stacking alternate arrays in the third direction. It is still possible, all be it more difficult, for a stable CSLL phase to exist. This phase is characterized by an isotropic in-plane conductivity $\sigma_{\|} \sim T^{-\alpha_{\|}}$and a conductivity $\sigma_{c} / a \sim T^{\alpha_{c}}$ in the direction perpendicular to the planes of the wires. Thus the conductivity along the planes is much larger than the perpendicular conductivity.

The extension of the above analysis to a system of coupled Luttinger liquids where both charge and spin excitations are gapless is straightforward. On each wire, there is a Luttinger liquid for charge and for spin. To maintain gapless Luttinger liquids and $S U(2)$ symmetry, we require that the spin degrees on each wire be represented by a Lagrangian of the form Eq. (2) with $\kappa_{s}=1$ and that at the fixed point there be no spin coupling between the wires. The fixed point for the charge degrees of freedom has the same form as for the gapped case. However, now, single-particle tunnelling as well as Josephson and
CDW couplings may be relevant. The phase diagram is quite complicated and will be discussed in a future publication. There is, however, a small but finite region of phase space where the sliding phase is stable.

In conclusion, we have demonstrated the existence of a non-Fermi metallic phase in two dimensions that maintains spin-charge separation and is stable to all pairwise potentials. This is a remarkable phase, which could be identified as a two-dimensional Luttinger liquid. We delay to a future publication the investigation of the stability of this phase with respect to all multiwire interactions.

RM and TCL acknowledge support from the National Science Foundation under grant DMR97-30405.

[1] V.J. Emery, in Highly conducting one-dimensional solids, edited by J. Devreese et al, (Plenum, New York, 1979). For a more recent review, see J. Voit, Rep. Prog. Phys. 58, 977 (1995).

[2] P.W. Anderson, Science 235, 1196 (1987).

[3] J.R. Engelbrecht, M. Randeria, and L. Zhang, Phys. Rev. B 45, 10136 (1992). Also, J.R. Engelbrecht and M. Randeria, Phys. Rev. Lett. 65, 1032 (1990).

[4] A. Houghton, J.H. Kwon, J.B. Marston, and R. Shankar, Journal of Physics-Condensed Matter 6, 4909 (1994).

[5] R.A. Klemm, and H. Gutfreund, Phys. Rev. B 14, 1086 (1976); L.P. Gorkov, and I.E. Dzyaloshinskii, Sov. Phys.JETP 40, 198 (1975). See also X.G. Wen, Phys Rev B 42, 6623 (1990).

[6] V.J. Emery, E. Fradkin, S.A. Kivelson, and T.C. Lubensky, cond-mat/001077.

[7] Ashwin Vishwanath and David Carpentier, condmat/0003036.

[8] L. Golubovic and M. Golubovic, Phys. Rev. Lett. 80, 4341 (1998). Erratum ibid. 81, 5704 (1998). C.S. O'Hern and T.C. Lubensky, ibid 80, 4345 (1998).

[9] C.S.O'Hern, T.C. Lubensky and J.Toner, Phys. Rev. Lett. 83, 2745 (1999).

[10] A related model had been considered in the context of cuprate superconductors by F. Guinea and G. Zimanyi, Phys. Rev. B 47, 501 (1993). See also A.H. Castro Neto and F. Guinea, Phys. Rev. Lett. 80, 4040 (1998). This model, however, includes only a subset of interactins. It does not consider interwire current and density interactions critical for establishing the existence of the CSLL phase.

[11] See J. Tranquada et al., Nature 375, 561 (1995); J. Tranquada, Physica B 241-243, 745 (1997).

[12] There has been some inconsistency in the literature regarding the definitions of $\theta$ and $\phi$. In this paper, we follow the notation of [6], and define $\theta$ as the phase variable, and $\phi$ as the conjugate density variable.

[13] V.J. Emery et. al, Phys. Rev. B 56, 6120 (1997).

[14] A. Luther and I. Peschel, Phys. Rev. Lett. 32, 922 (1974). See also, T. Giamarchi and H. Schulz, Phys. Rev. B 37, 325 (1998). 